\title{
i
}

\section{Note sulla storia della ricezione della poesia italiana in Brasile Lucia Wataghin}

\begin{abstract}
Note sulla ricezione in Brasile di Petrarca, Tasso e Metastasio, nel Settecento, e sulle traduzioni di Ariosto, Tasso, Leopardi, Ungaretti, Montale e altri nel Novecento.

PAROLE CHIAVE; traduzione; ricezione; poesia; Brasile; Italia.
\end{abstract}

È forse normale che nella grande famiglia costituita dalla poesia nelle diverse lingue europee i testi attraversino nei secoli mari e confini anche in mancanza di traduzioni: e infatti la scarsezza delle traduzioni in lingua portoghese non ha impedito una certa diffusione, almeno fra i poeti e gli studiosi, della poesia italiana in Brasile. Dante e Petrarca, Ariosto, Tasso e Metastasio, Leopardi e Foscolo si alternano o convivono, letti in lingua originale - o tradotri direttamente dai loro illustri lettori - nel corso dei secoli, nella memoria poetica dei letterati brasiliani. Gonçalves Dias traduce Dante, Petrarca, Metastasio e Foscolo; Alphonsus de Guimaraens si richiama a Petrarca e traduce l'ormai quasi dimenticato Stecchetti; Machado de Assis traduce il canto XXV dell' Inferno e cita la Vita Nuova e Petrarca, Leopardi e Tasso; gli arcadi brasiliani traducono Metastasio e citano Petrarca e Tasso. É noto che il capolavoro della prosa del Novecento brasiliano, Grande sertão: veredas, contiene numerose citazioni della Divina Commedia. Sono solo alcuni esempi che segnalano l'esistenza di rapporti fra la letteratura italiana e quella brasiliana - rapporti la cui effettiva portata è ancora da valutare. Limitandoci solo alla poesia, un primo rapido sondaggio 
nel campo delle traduzioni oggi disponibili in portoghese rivela un'unica presenza molto intensa - quella di Dante - e molte assenze nelle biblioteche e nel mercato editoriale brasiliano. Queste note si concentrano in particolare sul Settecento - il periodo in cui l'influenza italiana è più forte e diretta -, ma presentano anche alcuni dati sulle traduzioni della poesia italiana in Brasile nel Novecento.

Un caso notevole di influenza rilevante e indipendente dall'esistenza di traduzioni è quello di Petrarca, la cui unica traduzione brasiliana è parziale e relativamente recente ${ }^{1}$. Nel contesto della poesia portoghese dal Cinquecento a oggi, è noto che Petrarca occupa una posizione centrale - particolarmente grazie alla sua influenza su Camóes². Secondo lo studioso portoghese Vasco Graça Moura, che ha tradotto integralmente sia la Divina Commedia che il Canzoniere, Petrarca è l'autore più influente in assoluto sulla poesia portoghese: "Dante ha contribuito [alla poesia portoghese] solo con alcuni temi $\mathrm{e}$, più tardi, con alcuni brani dell'Inferno che attraevano l'immaginazione romantica, tormentata e cauchemardesque. Ma la verità è che, di quei cinque nomi [Dante, Petrarca, François Villon, Ronsard e Shakespeare], solo quello di Petrarca si eleva come grande nume tutelare di molta poesia portoghese, soprattutto nel secolo XVI [...]"3. Eppure, anche la letteratura portoghese non ha potuto contare su una traduzione integrale del Canzoniere fino ad anni recentissimi.

E probabilmente vero, come ha scritto Octavio Paz, che quasi tutta la poesia europea di amore può essere vista come una serie di glosse, variazioni e trasgressioni del Canzoniere di Petrarca. Possiamo ritenere che la sua presenza - diretta o

1. O Cancioneiro de Petrarca, trad. di Jamil Almansur Haddad, Rio de Janeiro/Săo Paulo, Livraria José Olympio Ed., 1945.

2. V. per esempio Rita Marnoto, "Petrarca em Porrugal. Ad eorum littus irem", in Petrarca 700 anos, Coimbra, Instituto de Estudos Italianos, Faculdade de Letras da Universidade de Coimbra, 2005.

3. Vasco Graça Moura, "Traduzir Petrarca", in Petrarca 700 anos, op. cit., p. 53. La traduzione integrale del Canzoniere, con il titolo As Rimas de Petrarca (2003), ha ricevuto il premio internazionale Diego Valeri. Per le traduzioni integrali della Divina Commedia e della Vita Nuova (1995), Graça Moura ha ottenuto il premio Pessoa. 
indiretta - in tutta la poesia occidentale sia tanto pervasiva che persiste, con o senza traduzioni. Cosi come nella letteratura portoghese, anche nella letteratura brasiliana Petrarca entra attraverso i poeti che l'hanno letto e ne hanno subito l'influenza.

Esistono studi importanti e dettagliati sul petrarchismo portoghese - soprattutto da Sá de Miranda, António Ferreira e Camões al manierismo ${ }^{4}$-, ma non si può dire lo stesso del petrarchismo brasiliano5 e perciò i dati qui presentati sono semplici indicazioni di possibili campi di ricerca.

Il petrarchismo ha assunto nei secoli valori e forme nuovi e diversi rispetto all'opera di Petrarca ed è naturalmente legato (particolarmente nella poesia barocca) ${ }^{6}$ anche ai diversi modi della trasgressione del modello petrarchista: in Brasile basterà pensare al più radicale dei trasgressori barocchi, Gregório de Matos, che può essere definito, come Quevedo, "misógamo, putañero y petrarquista"7 Nel senso che la sua "libido aggressiva" si libera dal controllo morale e formale della tradizione cortigiana, in modi che si contrappongono direttamente alle forme petrarchiste.

Il petrarchismo è una forte influenza anche nel Settecento dell'Arcadia, il periodo di massimo contatto fra la poesia brasiliana e quella italiana. E indubbio che l'ampia e importante produzione di poesia arcadica in Brasile è fondamentalmente debitrice alla tradizione poetica italiana, da Petrarca a Tasso. L'Arcadia brasiliana si ispira direttamente alle Accademie dell'Arcadia italiana (la prima delle quali, quel-

4. Rita Marnoto, O petrarquismo português do Renascimento e do Maneirismo, Coimbra, Acta Universitatis Conimbrigensis, 1997. V. anche i saggi raccolti nella sezione "Percursos do Petrarquismo na penf́nsula ibérica", in Petrarca 700 anos, op. cit.

5. L'ottimo studio di Luis André Nepomuceno, A musa desnuda e o poeta timido. O petrarquismo na Arcádia brasileira, São Paulo, Annablume; Patos de Minas/MG, Unipam, 2002, riguarda solo il periodo dell'Arcadia.

6. Come è noto, soprattutto nella poesia barocca, accanto al petrarchismo si sviluppa un filone anti-petrarchista, "veicolo di propositi satirici e stravaganti". V. Rita Marnoto, op. cit., p. 269. A questo proposito, vedi anche la sezione "Petrarquismo e antipetrarquismo", in Lufs André Nepomuceno, op. cit., pp. 67-81.

7. José Miguel Wisnik, "Introdução" a Gregório de Matos, Poemas Escolhidos, São Paulo, Cultrix, 1992, p. 24. L'espressione è di Octavio Paz, che la usa a proposito di Quevedo. 
la romana, fu fondata nel 1690), oltre che all'Arcadia quattrocentesca di Jacopo Sannazzaro e al Pastor Fido di Guarini. Ma mentre l'Arcadia settecentista italiana si risolve in un classicismo "minore e ornamentale" e in un" arte di intrattenimento e di evasione", l'Arcadia brasiliana conta alcuni grandi poeti. Il maggiore poeta dell'Arcadia, il luso-brasiliano T. A. Gonzaga, è autore di uno dei più bei canzonieri della tradizione occidentale, le lire dedicate a Marilia - di chiara ascendenza petrarchista. Petrarca è citato direttamente nella lira XLV', in cui il poeta esorta Marilia a disprezzare le ricchezze materiali e apprezzare invece il fatto che la sua poesia le darà l'immortalità, dichiarando allo stesso tempo la propria appartenenza alla linea illustre Petrarca/Tasso:

Se não houvesse Tasso, nem Petrarca,

Por mais que qualquer delas fosse linda,

Já não sabia o mundo se existiram

nem Laura, nem Clorinda.

Tasso, soprattutto con la favola pastorale l'Aminta (1573), è un punto di riferimento fondamentale per il canzoniere di Gonzaga e per tutti i poeti dell'Arcadia. Numerosi riscontri testuali attestano la presenza di Petrarca e Tasso nell'opera di Basílio da Gama, l'arcade brasiliano di cui Machado de Assis scrisse che "nessun altro, nella nostra lingua, ha avuto [un'arte] più armoniosa e pura"10. Antonio Candido ${ }^{11}$ e soprattutto Sérgio Buarque de Hollanda ${ }^{12}$ presentano numerosi esempi di parafrasi e citazioni quasi letterali (tradotte in versi in portoghese) dei Trionfi di

8. Romano Luperini et alii, La scrittura e l'interpretazione, vol II, tomo I, p. 269.

9. Tomás António Gonzaga, Obras completas, a cura di Rodrigues Lapa, São Paulo/Rio de Janeiro/Recife/Porto Alegre, Companhia Editora Nacional, 1942.

10. Apud Sérgio Buarque de Holanda, Capitulos de literatura colonial, São Paulo, Brasiliense, 1991-2000, p. 133.

11. Antonio Candido, Formạ̧äo da literatura brasileira, Belo Horizonte, Ed. Itatiaia, 1997 (1. ed. 1975), p. 123.

12. Sérgio Buarque de Holanda, "A Arcádia heróica", op. cit., p. 137 e segg. 
Petrarca e della Gerusalemme di Tasso nell' Uraguai di Basílio da Gama. Secondo Buarque de Holanda, il lirismo di Basílio da Gama ha origine nel "supposto 'romanticismo' o 'barocchismo' fondamentale di Tasso"13 e l'Uraguai ha in comune con la Gerusalemme "la costante invasione del lirismo nell'epica" ${ }^{14}$.

Ancora nel quadro dei rapporti degli arcadi brasiliani con la poesia italiana, registriamo l'intensa presenza di Pietro Metastasio. Fra gli inediti di Cláudio Manuel da Costa si trovano varie composizioni teatrali chiaramente ispirate a Metastasio, di cui l'arcade brasiliano tradusse anche numerosi melodrammi. Ė noto che Cláudio Manuel da Costa, grande sonettista, "il più arcade degli arcadi brasiliani"'s, fu profondamente influenzato da Petrarca e Sannazzaro e mantenne stretti legami con l'Italia: scrisse tra l'altro 14 sonetti di ispirazione metastasiana ${ }^{16}$ in italiano (alcuni dei quali sono stati recentemente tradotti in portoghese da Pedro Garcez Ghirardi). Forti influenze di Mestastasio si riscontrano anche nelle opere di altri arcadi brasiliani, come Santa Rita Durão, che visse in Italia per più di vent'anni, e si ispirò anche a Ariosto e Tasso ${ }^{17}$, e Silva Alvarenga.

Il prestigio di Metastasio in Brasile (come del resto anche in Europa) fu immenso $^{18}$ È noto che l'opera di Pietro Metastasio ebbe un ruolo determinante nella

13. Ib., p. 142.

14. Ib., p. 141.

15. La definizione è di José Veríssimo, apud Sérgio Buarque de Holanda, op. cit., p. 269.

16. Alfredo Bosi, História concisa da literatura brasileira, Sáo Paulo, Cultrix, 1984, p. 69.

17. Ib., p. 108 e segg.

18. Un prestigio "quasi impareggiabile", secondo Buarque de Holanda, op. cit., p. 125. V. anche Carla Inama, Metastasio e i poeti arcadi brasiliani, São Paulo, USP/FFLCH, Boletim 231, 1961. Le traduzioni dei melodrammi di Metastasio a opera di Cláudio Manuel da Costa - conservate dall'autore in manoscritri - sono di difficile reperibilità: si trovano forse ora, almeno in parte, nel volume Obras, pubblicato a Coimbra nel 1768 (V. Sérgio Buarque de Holanda, op. cit., p. 250). Nella Biblioteca Nacional di Rio de Janeiro esistono copie di 4 opere di Metastasio, musicate nella corte portoghese, con testo bilingue. Il catalogo della Biblioteca Nacional registra anche la traduzione di Bocage dell'opera Régulo e la traduzione "segundo o estillo do Theatro portuguez por Nicoláo Luiz da Sylua", dell'opera Ezio, entrambe di Metastasio. 
storia del melodramma, e fu amata anche da Voltaire e da Rousseau. Quest'ultimo tradusse in francese la canzone "La libertà", che destò l'interesse di vari poeti brasiliani contemporanei: Antonio José da Silva ne adottò tema e toni in un brano dell' Esopaida, Alexandre de Gusmão e Basílio da Gama la tradussero in portoghese, Cláudio Manuel da Costa ne fece una parodia ${ }^{19}$. Il poeta celebra la fine dell'incantesimo amoroso, la liberazione dai lacci dell'amore; l'amante si dichiara disamorato, ma alla fine lo stratagemma è palese: si trattava di un'irosa, ulteriore confessione d'amore. Ecco il bel finale, nella traduzione di Basílio:

Perdes por incostante

$\mathrm{O}$ amor mais verdadeiro;

Não sei de nós primeiro

Quem se há de consolar.

Eu sei que um firme amante

Não se acha a toda a hora

Uma alma enganadora

É fácil de encontrar. ${ }^{20}$

Uno dei più noti intermediari fra il mondo erudito e quello popolare, nel Settecento brasiliano, è un conoscitore e traduttore di Metastasio, Domingos Caldas Barbosa. Caldas Barbosa è autore della Viola de Lereno (1798), una raccolta di lunduns e modinhas brasiliani adattati al linguaggio dell'Arcadia, "un caso tipico di contaminatio della tradizione orale, parlata e cantata, con il linguaggio erudito"21. Carla Inama riporta ${ }^{22}$ una sua "traduzione e glosa" di una poesia di Metastasio, "La partenza" (1746). Più che di una traduzione, si tratta di una libera imitazione, che sacrifica le note psicologiche più complesse della graziosa canzone originale,

19. Sérgio Buarque de Holanda, op. cit., p. 195 e segg.

20. I testi completi della canzone merastasiana e della traduzione di Basilio da Gama si trovano in Carla Inama, op. cit., pp. 101-107.

21. Alfredo Bosi, op. cit., p. 88.

22. Carla Inama, op. cit., p. 111. 
di cui mantiene solo il tema generale (il lamento per la separazione dall'amata, in Metastasio un "barbaro addio funesto") e il ritornello, che si ripete 7 volte: " $E$ tu chi sa se mai/Ti sovverrai di me!", che Caldas Barbosa traduce: "Ab! Quem sabe se LerenolInda a ver-te tornará"

Attraverso Caldas Barbosa e altri poeti arcadi, l'esempio di Metastasio, con la sua grande forza comunicativa e accessibilità universale (per l'epoca), con la sua musicalità e abilità nel creare forti effetti psicologici, ha un peso anche sulla lirica popolare brasiliana. Si è detto giustamente che la modinha brasiliana - che sorge in parte proprio dalla poesia arcadica portoghese e brasiliana ${ }^{23}-\mathrm{c}$ anche un'erede dell'arietta metastasiana ${ }^{24}$.

Nel Novecento, grazie anche ad alcune presenze significative a São Paulo (vi risiedono a lungo per esempio il poeta Giuseppe Ungaretti e lo studioso e brillante tradurtore italiano di Guimaráes Rosa, Edoardo Bizzarri), i rapporti fra la cultura italiana e quella brasiliana si intensificano nuovamente. Cresce, naturalmente grazie allo sviluppo dell'editoria, il numero di traduzioni dall'italiano, soprattutto di opere di narrativa e saggi, ma anche di poesia ${ }^{25}$. Dante è in assoluto il poeta più

23. Th. Braga, Filinto Elysio e os dissidentes da Arcddia, apud L. A. Nepomuceno, op. cit., p. 212.

24. Carla Inama, op. cit., p. 85.

25. Fra le traduzioni recenti segnaliamo la prima, ed eccellente, traduzione (premiata con il jabuti) di 8 canti del Furioso e alcuni brani di altri canti, di Pedro Garcez Ghirardi [Ludovico Ariosto, Orlando Furioso, trad. introd. e note di Pedro Garcez Ghirardi, São Paulo, Ateliê, 2003]. Dobbiamo a Marco Lucchesi l'edizione dell'esemplare traduzione della Gerusalemme Liberata, a opera del portoghese José Ramos Coelho, pubblicata nel 1864 e rivista e corretta dal traduttore nel 1905 [Torquato Tasso, Jerusalém Libertada, trad. José Ramos Coelho, introduzione e note di Marco Lucchesi, Rio de Janeiro, Topbooks, 1998]. Entrambe le edizioni sono arricchite da note e commenti dei traduttori e/o editori. Marco Lucchesi ha anche curato l'imponente antologia di poesia e prosa leopardiana [Giacomo Leopardi, Poesia e prosa, Rio de Janeiro, Nova Aguilar, 1996], importante anche per l'ottima sezione dedicata alla fortuna critica, che contiene 28 saggi (di cui 6 brasiliani), selezionati fra i più significativi sull'opera di Leopardi. Per la parte poetica l'edizione, purtroppo non bilingue, raccoglie le traduzioni di Affonso Félix de Souza, Alexei Bueno, Álvaro Antunes, Ivan Junqueira, Ivo Barroso, José Paulo Paes. Fra le traduzioni italiane di José Paulo Paes ricordiamo anche i sonetri lussuriosi di Aretino 
tradotto $^{26}$. Sia Dante che Petrarca hanno avuto una rilevanza enorme nella tradizione occidentale (benché con fasi alterne nel favore dei lettori e della critica), e quindi anche - direttamente o indirettamente - sulla poesia brasiliana. É noto che dantismo e petrarchismo sono due principi, due modi di fare poesia che la critica italiana - da De Sanctis a Contini alla critica contemporanea - ha usato e tuttora usa per spiegare due diverse macrotendenze della letteratura italiana. In questa dicotomia, Dante rappresenta grossomodo l'apertura, l'espansione - il plurilinguismo e il pluritematismo -, mentre Petrarca rappresenta la chiusura, la selezione formale, linguistica e tematica - il monolinguismo e il monotematismo. La "vocazione per il reale"27 - più dantesca che petrarchesca - e un'ansia di espressività più radicale, una tendenza allo sperimentalismo nelle forme, all'espansione del lessico e, più in generale, quello che si chiama l'espressionismo" dantesco risponde forse meglio alle necessità o al temperamento dei poeti del Novecento, ma la linea petrarchista continua ad alternarsi o a sovrapporsi a quella dantista nelle varie fasi della letteratura e spesso anche nelle opere di singoli autori. Per fare un solo esempio italiano, un poeta come Montale, considerato senz'altro "dantista", presenta almeno una fase da lui stesso definita "petrarchista" in cui, petrarchescamente, il poeta si rivolge

[Pietro Aretino, Sonetos luxuriosos, trad. introd. e note di José Paulo Paes, Săo Paulo, Companhia das Letras, 2000], con introduzione del traduttore, e un sonetro di Pietro Bembo [José Paulo Paes, Gaveta de tradutor. Versóes de poesia. Florianópolis, Letras Contemporâneas, 1996]. Non si ha notizia di traduzioni di Foscolo.

26. La Divina Commedia è stata tradotta integralmente dal Barão da Vila da Barra (1888), Xavier Pinheiro (s/d), Cristiano Martins (1976), ftalo Mauro (1998), per citare le principali traduzioni integrali in versi. Machado de Assis ha tradotto un canto dell'Infemo, Eduardo Guimaraens un canto dell'Inferno, Haroldo de Campos ha tradotto parti delle Rime e della Vita Nova e 6 canti del Paradiso; Augusto de Campos 4 canti dell'Inferno e due del Purgatorio; Decio Pignatari la Vita Nuova; Henriqueta Lisboa 14 canti del Purgatorio e tre sonetti della Vita Nuova; Jorge Wanderley ha tradotto parti delle Rime e della Vita Nuova. Alle traduzioni di Dante, Haroldo e Augusto de Campos hanno affiancato traduzioni di Guinizzelli e Cavalcanti.

27. L'espressione "vocaf̧ão para o real" è di Alfredo Bosi, che la usa per definire una delle tendenze più profonde della poesia contemporanea, che accomuna poeti come Pound e Montale, Ponge e Drummond, Murilo e Cabral de Melo Neto. Cf. Alfredo Bosi, op. cit., p. 504. 
a una donna astratta come l'aria: "donna o nube, angelo o procellaria"n. In Brasile (come anche in Italia) il modello dantesco è senz'altro preferito dalle avanguardie, che hanno offerto eccellenti traduzioni e commenti di testi di Dante - ma nella poesia brasiliana troviamo anche accenni (talvolta polemici) a Petrarca.

Una delle poesie più famose di Carlos Drummond de Andrade - No meio do caminho tinha uma pedra - inizia con una citazione del primo verso della Commedia. Nell'incontro fra poesia antica e moderna l'ostacolo, nel mezzo del cammin, si trasforma: non è più una "selva oscura", ma una prosaica, dura pietra. Le più chiare influenze dantesche (ma anche camoniane e quindi petrarchiste) si riscontrano in Claro Enigma, il libro che segna il ritorno di Drummond a metrica e forme classiche. Nei versi del sonetto Oficina irritada, per esempio:

Eu quero compor um soneto duro

Como poeta algum ousara escrever.

Eu quero pintar um soneto escuro

Seco, abafado, dificil de ler.

si può riconoscere un riferimento alle Rime Petrose dantesche (Così nel mio parlar voglio esser aspro). "A máquina do mundo", sesta parte del libro, ricorda Dante "per il suo andamento narrativo, la gravità e il contenuto cosmico-esistenziale", oltre che per la metrica (terzine non rimate), e anche la poesia che chiude il volume, Relogio do rosário, rivela influenze dantesche ${ }^{29}$. Pare completamente non-petrarchista, per la mescolanza degli stili, la prevalenza di uno stile umile e dimesso e la passione per la concretezza anche tutta la sezione dell'Antologia poética di Drummond, il cui titolo amar-amaro allude però al petrarchismo (per la precisione, la formula "amare senza amaro non si può" ̀े del primo dei petrarchisti, Pietro Bembo).

28. È la fase delle poesie di Finisterre, come afferma lo stesso Montale in "Intervista immaginaria"(1946). Apud Giulio Ferroni, op. cit., p. 88.

29. Francisco Achcar, Carlos Drummond de Andrade, São Paulo, PubliFolha, 2000, p. 82 e segg. 
Il poeta italiano contemporaneo più noto in Brasile è Ungaretti. Il libro più tradotto di Ungaretti è L'Allegria (integralmente da Sérgio Wax e Geraldo Holanda Cavalcanti, e parzialmente da Haroldo de Campos e Henriqueta Lisboa), ma c'è anche una bella traduzione di Aurora Bernardini de Il Dolore, il libro più "brasiliano" di Ungaretti. ${ }^{30}$ È noto che Ungaretti è il poeta italiano più caro alle avanguardie pauliste, e si potranno forse trovare alcune tracce della sua influenza in alcuni poeti brasiliani (Murilo Mendes, per esempio, ha dichiarato di sentirsi "più vicino a Ungaretti che ad altri poeti più istintivi italiani”). Ma la poesia modernista brasiliana - nella misura in cui è antilirica, discorsiva, realistica, concreta, prosastica - è distante dalla rarefazione e estrema astrattezza di gran parte della poesia di Ungaretti. Come nota Bosi, sono piuttosto i crepuscolari Gozzano e Corazzini, con il loro stile malinconico e dimesso, a lasciare tracce sulla poesia dei maggiori modernisti brasiliani, come Bandeira e Mário de Andrade ${ }^{31}$ Gozzano e Corazzini sono all'origine, insieme a Saba, di un'importante linea della poesia italiana, che Pasolini defini "antinovecentista" e che tende all'abbassamento dello stile e della lingua e all'avvicinamento alla prosa.

In Brasile, la tendenza a privilegiare una poesia più realistica e vicina alla prosa è in parte confermata negli ultimi anni da alcune scelte dei traduttori: più di due terzi del volume di traduzioni di Montale di Geraldo Holanda Cavalcanti ${ }^{32}$ sono dedicati all'ultima fase di Montale (le poesie scritre dal '71 in poi, da Satura, Diari

30. Le traduzioni brasiliane delle poesie di Ungaretti: una selezione di 13 liriche da L'Allegria e da Il Sentimento del Tempo tradotte da Henriqueta Lisboa, in Poesia traduzida, Belo Horizonte, Ed. UFMG, 2001; L'Allegria. ed. bilingue, trad. Sérgio Wax, Belém, CEJUP, 1992; Daquela estrela à outra, trad. di Haroldo de Campos [da L'Allegria, Il Sentimento del Tempo, Il Taccuino del Vecchio, Derniers Jours] e Aurora F. Bernardini [Il Dolore], org. e introd. di Lucia Wataghin, São Paulo, Ateliê Editorial, 2003; L'Allegria, trad. di Geraldo Holanda Cavalcanti, prefazione di Luciana Stegagno Picchio, Rio de Janeiro/Sāo Paulo, Record, 2003.

31. Alfredo Bosi, op. cit., p. 300.

32. Eugenio Montale, Poesias, trad. Geraldo Holanda Cavalcanti, prefazione di Luciana Stegagno Picchio, Rio de Janeiro/São Paulo, Record, 1997. Tra le traduzioni di Cavalcanti, ricordiamo anche Poesias, di Salvatore Quasimodo, prefazione di Luciana Stegagno Picchio, Rio de Janeiro/São Paulo, Record, 1999. 
e Quaderni), una dimostrazione di chiara preferenza per il Montale più prosastico, diaristico e discorsivo. Sono attualmente in preparazione una serie di traduzioni importanti di due poeti antilirici, programmaticamente distanti dalla poesia "pura": Umberto Saba (ormai considerato uno dei più grandi del Novecento) (traduzione di Holanda Cavalcanti) e Cesare Pavese (traduzione di Maurício Santana Dias), ma anche quelle di un terzo poeta di valore, atipico, ma fondamentalmente lirico, Dino Campana (traduzione di Aurora Bernardini). Sono da segnalare infine, le traduzioni di Henriqueta Lisboa dell'Infinito di Leopardi e di varie poesie di Pavese $e^{33}$ e le pochissime, ma eccellenti traduzioni di Nelson Ascher di Ungaretti e Montale ${ }^{34}$.

Lirici o antilirici, non importa. La recente intensificazione delle traduzioni di poesia italiana è certamente benvenuta e ci auguriamo che anche altri poeti italiani - da Gozzano a Penna, da Luzi a Giudici, da Sereni a Caproni a Bertolucci - siano presto tradotti e messi a disposizione del pubblico brasiliano.

RESUMO: Notas sobre a recep̧äo no Brasil de Petrarca, Tasso e Metastasio (no seculo XVIII) e sobre as traduç̄es de Ariosto, Tasso, Leopardi, Ungaretti, Montale e outros no século XX.

PALAVRAS-CHAVE: traduçāo; recep̧ẫo; poesia; Brasili Itália.

33. Henriqueta Lisboa, op. cit.

34. Nelson Ascher, Poesia Alheia, 124 poemas traduzidos, Rio de Janeiro, Imago, 1998 (4 poesie di Ungaretti) e Revista de Italianistica XII (una poesia di Montale). 